\title{
Quantum Dots for In Vivo Small-Animal Imaging
}

\author{
Laurent A. Bentolila ${ }^{1,2}$, Yuval Ebenstein ${ }^{1}$, and Shimon Weiss ${ }^{1-3}$ \\ ${ }^{I}$ Department of Chemistry and Biochemistry, UCLA, Los Angeles, California; ${ }^{2}$ California NanoSystems Institute, UCLA, \\ Los Angeles, California; and ${ }^{3}$ Department of Physiology, UCLA, Los Angeles, California
}

Nanotechnology is poised to transform research, prevention, and treatment of cancer through the development of novel diagnostic imaging methods and targeted therapies. In particular, the use of nanoparticles for imaging has gained considerable momentum in recent years. This review focuses on the growing contribution of quantum dots (QDs) for in vivo imaging in smallanimal models. Fluorescent QDs, which are small nanocrystals $(1-10 \mathrm{~nm})$ made of inorganic semiconductor materials, possess several unique optical properties best suited for in vivo imaging. Because of quantum confinement effects, the emission color of QDs can be precisely tuned by size from the ultraviolet to the near-infrared. QDs are extremely bright and photostable. They are also characterized by a wide absorption band and a narrow emission band, which makes them ideal for multiplexing. Finally, the large surface area of QDs permits the assembly of various contrast agents to design multimodality imaging probes. To date, biocompatible QD conjugates have been used successfully for sentinel lymph node mapping, tumor targeting, tumor angiogenesis imaging, and metastatic cell tracking. Here we consider these novel breakthroughs in light of their potential clinical applications and discuss how QDs might offer a suitable platform to unite disparate imaging modalities and provide information along a continuum of length scales.

Key Words: quantum dots; molecular imaging; optical imaging; PET; nanoparticles; near-infrared fluorescence (NIRF) imaging; nanotechnology; cancer; nanomedicine

J Nucl Med 2009; 50:493-496

DOI: 10.2967/jnumed.108.053561

Molecular imaging (MI) encompasses various noninvasive techniques such as MRI and magnetic resonance spectroscopy, optical bioluminescence and fluorescence imaging, ultrasound, SPECT, and PET. MI, which is used to visualize, characterize, and measure biologic processes at the cellular and molecular levels in living organisms, has the potential to markedly enhance both diagnosis and management of disease. When combined with an anatomic imaging modality such as CT, PET/CT and SPECT/

Received Dec. 2, 2008; revision accepted Jan. 28, 2009.

For correspondence or reprints contact: Laurent A. Bentolila, UCLA, 607 Charles E. Young Dr. East, Los Angeles, CA 90095.

E-mail: Ibento@chem.ucla.edu

COPYRIGHT @ 2009 by the Society of Nuclear Medicine, Inc.
CT can help characterize the molecular status of tumors deep within living animals and cancer patients. The use of radionuclide tracers, however, entails complex in situ fabrication processes inherent to the short half-life of most isotopes administered in clinical settings and requires careful handling because of patient safety issues and radioactive waste disposal. Radioactivity-based methods are also limited to a single detectable physical observable (i.e., no simultaneous multiplexing possible, although sequential imaging of a second radiopharmaceutical after the first has decayed is still achievable), and they lack the high spatial resolution required to monitor the earliest manifestation of disease at the cellular level (1-100 $\mu \mathrm{m})$. Deep-tissue multiphoton microscopy, on the other hand, can image fluorescently tagged living cells in 3 dimensions with high sensitivity (down to the single-molecule level), with high spatial (submicrometer) and temporal (millisecond) resolution. However, optical imaging suffers from 2 major drawbacks: the limited penetration depth of light in living tissue (a few centimeters, at best, in the near-infrared [NIR] wavelength between 675 and $900 \mathrm{~nm}$ ) and the insufficient brightness and stability of commonly used organic fluorophores and genetically encoded fluorescent proteins. Moreover, the toxic radicals and photoproducts generated on repeated excitation or photodestruction of dyes usually prevent long-term in vivo imaging.

Recent advances in nanotechnology offer some prospects to combine the best of each imaging technique with respect to sensitivity and specificity. There is now a vast array of artificial particulate systems used as diagnostic agents capable of targeting different cells in vivo. Those include colloidal gold, superparamagnetic iron-oxide crystals, dendrimers, polymeric micelles and liposomes, nanotubes, nanowires, nanoshells, and quantum dots (QDs), to name just a few (1).

This article focuses on the use of QDs for in vivo imaging. As a short primer on this subject, we explain what QDs are; their demonstrated use in molecular imaging, which has so far been directed primarily toward the study of cancer and cancer-related small-animal models; their potential clinical applications (if any); and how QDs might offer a powerful platform to unite all the disparate imaging modalities to encompass the macro- to the nanoscale.

\section{QDs IN A NUTSHELL}

The term quantum dot describes a material in which charge carriers (electrons and holes) are confined in all 3 dimensions. Because of their quantum nature, these charge carriers occupy a volume determined by the physical properties of the material in which they are contained (usually, up to a few tens of nanometers for semiconductors). Confinement occurs when a material is shrunk to dimensions smaller than 
this characteristic volume, and this in turn gives rise to new physical properties nonexistent in the bulk material. The most vivid demonstration of quantum confinement is the relationship between emission color of QD particles and their size.

Because the emission wavelength of QDs is size-dependent, fluorescence color may be fine-tuned throughout the visible and NIR spectrum during chemical synthesis, by controlling the QD size and composition. In addition, QDs have a continuous absorption band and a narrow, gaussian emission profile. These combined properties enable excitation of multiple-colored QDs with a common excitation source and the detection of multiple colors within a limited spectral window without cross-talk. Practically, a peak-to-peak wavelength separation of $25-50 \mathrm{~nm}$ is feasible.

Although considerably larger, QDs are brighter than are spectrally equivalent organic dyes under commonly used (nonsaturating) excitation power. Their high quantum yield in conjunction with a highextinction coefficient results in an exceptionally high-fluorescence photon flux. In addition, QDs are extremely photostable, making them ideally suited for long-term observations or repeated measurements. Finally, QDs exhibit long fluorescence lifetimes (tens of nanoseconds), which enable time-gated imaging that eliminates autofluorescence and enhances the sensitivity of fluorescence detection in vivo.

Semiconductor QDs are small inorganic crystals usually synthesized at high temperatures in nonpolar organic media. They must be rendered water-soluble via hydrophilic encapsulation before bioimaging. Various approaches have been reported including polymer coating typical of the commercial QDs from Invitrogen, peptide-coated QDs (2), and small thiol-containing ligands or amino acids $(3,4)$. These coatings also provide multiple reactive chemical groups for attaching functional targeting and therapeutic moieties and other contrast agents (e.g., radionuclides) to design multimodality probes that could potentially target, label, and treat.

\section{QDs IN MOLECULAR IMAGING AND CANCER MEDICINE}

The targeted delivery of biocompatible QD conjugates in vivo has been limited to date to tissue-specific vascular biomarkers. Gao et al. used QDs conjugated to monoclonal antibodies directed against prostate-specific membrane antigen to target and image human prostate cancer cells growing in live mice (Fig. 1A) (5). More recently, arginineglycine-aspartic acid peptide-conjugated QDs have been used to specifically target integrin $\alpha_{\mathrm{v}} \beta_{3}$ in a murine xenograft model $(6,7)$ because the integrin $\alpha_{v} \beta_{3}$ is significantly upregulated in tumors but not in normal tissues (Fig. 1B). Such studies raise unique possibilities for multiplexed optical imaging of molecular targets in vivo. Another important aspect to consider is that QDs can be tuned to emit in the NIR region of the spectrum, in which tissue autofluorescence is considerably reduced and excitation light penetration increased. For instance, the superiority of NIR QDs (emitting at $850 \mathrm{~nm}$ ) has been demonstrated in sentinel lymph node (SLN) mapping, a common procedure in breast cancer surgery, whereby the lymph node closest to the organ affected is monitored for the presence of locally disseminated cancer cells. After intradermal injection in live pigs and mice, NIR QDs allowed image guidance throughout the entire procedure, virtually free of any background. SLNs and their eventual removal were imaged in real time, without the need of traditional dyes or radioactive tracers (Fig. 1C). The optimal separation from autofluorescence background and the increased penetration of both excitation and light emission through thick tissues (about $1 \mathrm{~cm}$ below the skin surface) allowed the surgeon to minimize the size of the incision necessary to remove the incriminated SLN. This separation represents a significant breakthrough, suggesting that NIR imaging of QDs could possibly aid in rapidly and accurately
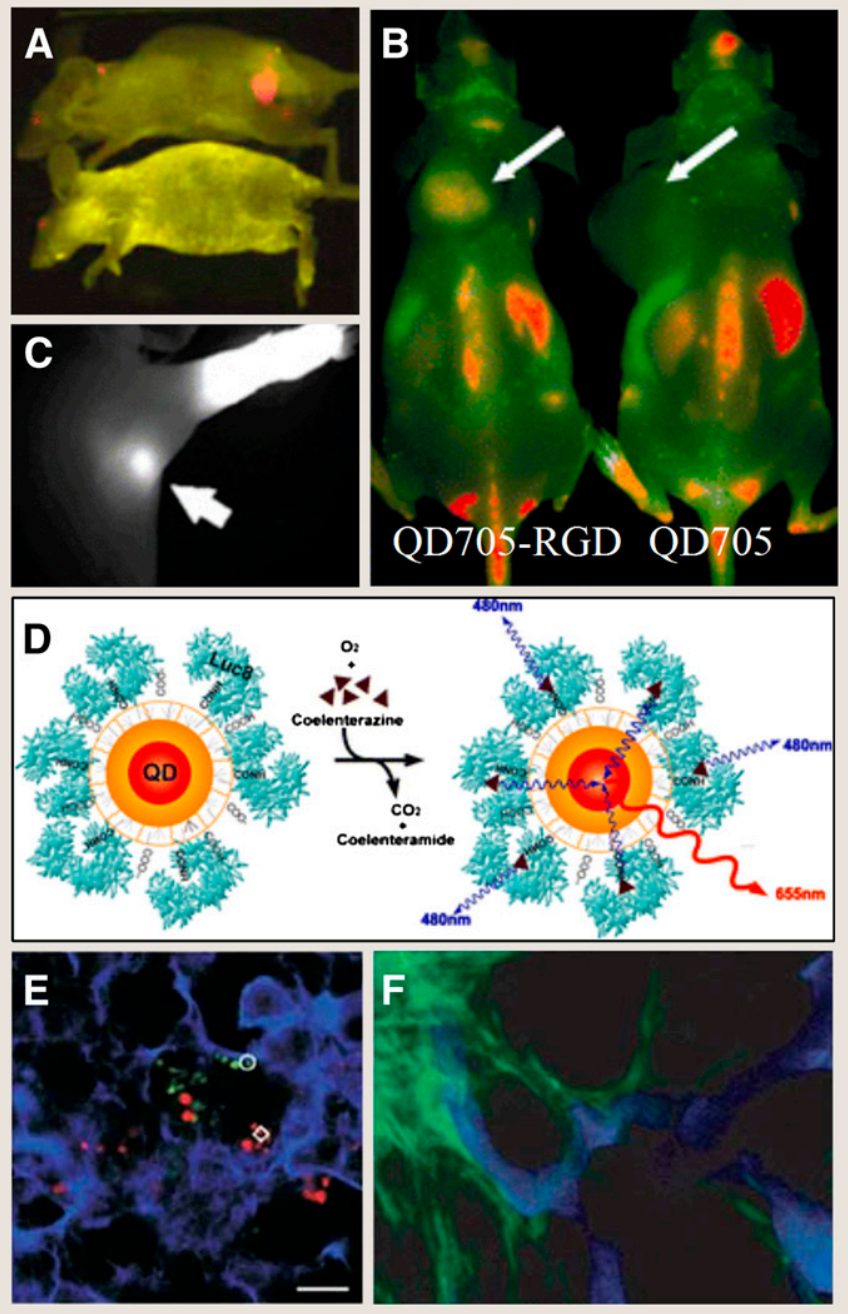

FIGURE 1. (A) Molecular targeting and in vivo imaging of prostate tumor using QD-antibody conjugate. (Reprinted with permission of (5).) (B) In vivo near-infrared fluorescence imaging of tumor-bearing mice injected with QD705-RGD peptide or QD705. (Reprinted with permission of (7).) (C) NIR QDs taken up by sentinel lymph nodes. (Reprinted with permission of (8).). (D) Principle of self-illuminating QDs through bioluminescence resonance energy transfer of Luc8 acting as donor to acceptor QDs on oxidation of coelenterazine. (Reprinted with permission of (10).) (E) Simultaneous tracking of different populations of QD-labeled metastatic tumor cells in mice lung tissue. (Reprinted with permission of (11).) (F) Intravital imaging provides clear separation of QD-labeled tumor vessels (blue) from green fluorescent protein-expressing perivascular cells (green). (Reprinted with permission of (12).)

locating, delineating, and removing SLNs or even possibly other types of cancerous lesions in humans $(8,9)$.

Despite those advantages of NIR emission, locating fluorescent objects in vivo can still be challenging because tissue absorption and scattering, in addition to limiting the light coming out of the subject, also limit the amount of incident excitation light that reaches the fluorophore. Bioluminescence resonance energy transfer, which converts chemical energy into light energy, has been used to circumvent this last issue. So et al. have used luciferase-conjugated QDs to obtain self-illuminating QDs that fluoresce without the need of an external 
illumination source (10). Upon binding to its substrate coelenterazine, luciferase emits broad-spectrum blue light sufficient to excite the bound QD (Fig. 1D). This bioluminescent QD technology totally eliminates the issue of tissue autofluorescence because no excitation light is required and maximizes high-penetrating NIR wavelengths for imaging. The net gain is an increased sensitivity detection that outperforms all current QD and NIR dyes in in vivo imaging. QD-labeled tumor cells have also provided the first means to study extravasation in vivo. Using a well-established model of cancer cell metastasis in mice, Voura et al. have demonstrated that it was possible to follow the distribution of B16 melanoma cells in resected tissues with single-cell sensitivity using multiphoton laser scanning microscopy (11). Spectral imaging of QDs allowed the simultaneous tracking of different QD-tagged populations of cells in their natural tissue environment and in the same animal (Fig. 1E). This new tool may contribute to our understanding of metastasis, which remains a fundamental barrier to the development of effective cancer therapies. Intravital microscopy of tumors labeled with QDs has also revealed the pathophysiology of tumors with unprecedented morphologic details by allowing the differentiation of the tumor blood vasculature from both the perivascular cells and the matrix (Fig. 1F) (12).

Despite the great potential of QD optical imaging, it is still difficult to quantify accurately any QD signal in deep tissues based on fluorescence alone. This intrinsic limitation is now being addressed by developing QD-based probes that combine multiple molecular imaging modalities onto a single QD nanoparticle platform. Indeed, the tens to hundreds of square nanometers of surface area of QDs represent invaluable assets for surface integration of paramagnetic or radioactive agents that enable 3 -dimensional tomography techniques. For example, 1,4,7,10-tetraazacyclododecane-1,4,7,10-tetraacetic acid (DOTA) conjugated to QDs enabled PET after chelation with ${ }^{64} \mathrm{Cu}$ $(6,13-15)$. Such dual-modality probes have provided unique quantitative information pertaining to tumor-targeting efficiency $(6)$ and in vivo kinetic biodistribution $(14,15)$ that is helping optimize the next generation of QD probes for in vivo imaging. The long-term goal of such efforts is the design of QD-based imaging agents that will allow simultaneous, quantitative PET detection of multiple spectrally distinct targets. Nuclear spin labels for MRI (16) or SPECT/CT contrast radionuclide (17) could also be incorporated into QDs. A further step could involve transmission electron microscopy imaging of the precise localization of QDs within cells and tissues (18). As such, QDs have the potential to provide information over a wide range of length scales.

\section{CURRENT CHALLENGES FACING MOLECULAR IMAGING WITH QUANTUM DOTS}

This handful of successful in vivo imaging and targeting studies reflects the current challenges facing QD technology, namely the relatively large size $(15-30 \mathrm{~nm})$ and short circulation half-life of QDs in the blood vascular system due to their rapid uptake and accumulation in the liver. Current efforts are geared toward prolonging the circulation time of QDs by attaching passivating molecules such as polyethylene glycol and by controlling the overall charge of the particles to prevent their adsorption to plasma proteins $(4,14,15)$. Clearance from the body is a prerequisite to the clinical use of any contrast agent. In conjunction with prolonging circulation time in the blood, similar parameters are being studied for their effect on clearance of QDs from the body, predominantly through the kidneys. An intriguing recent finding suggests a size threshold of 5-6 nm in diameter, below which the QDs cannot escape the liver and be cleared through the kidneys (19). In addition, because most QDs are composed of heavy metals known to be toxic in their soluble form, the potential cytotoxicity of those inorganic particles arises (20). One avenue is to avoid toxicity altogether, using nontoxic formulations of QDs such as $\mathrm{SiC}$ (21) or silicon (22). However, InAs-based particles seem closest to clinical applications because they combine the smaller sizes with the highest quantum yield in the NIR spectrum (23). Although a low dosage of arsenic is tolerated by cells, cadmium ions are among the most toxic heavy-metal contaminants; therefore, syn-

\section{A}
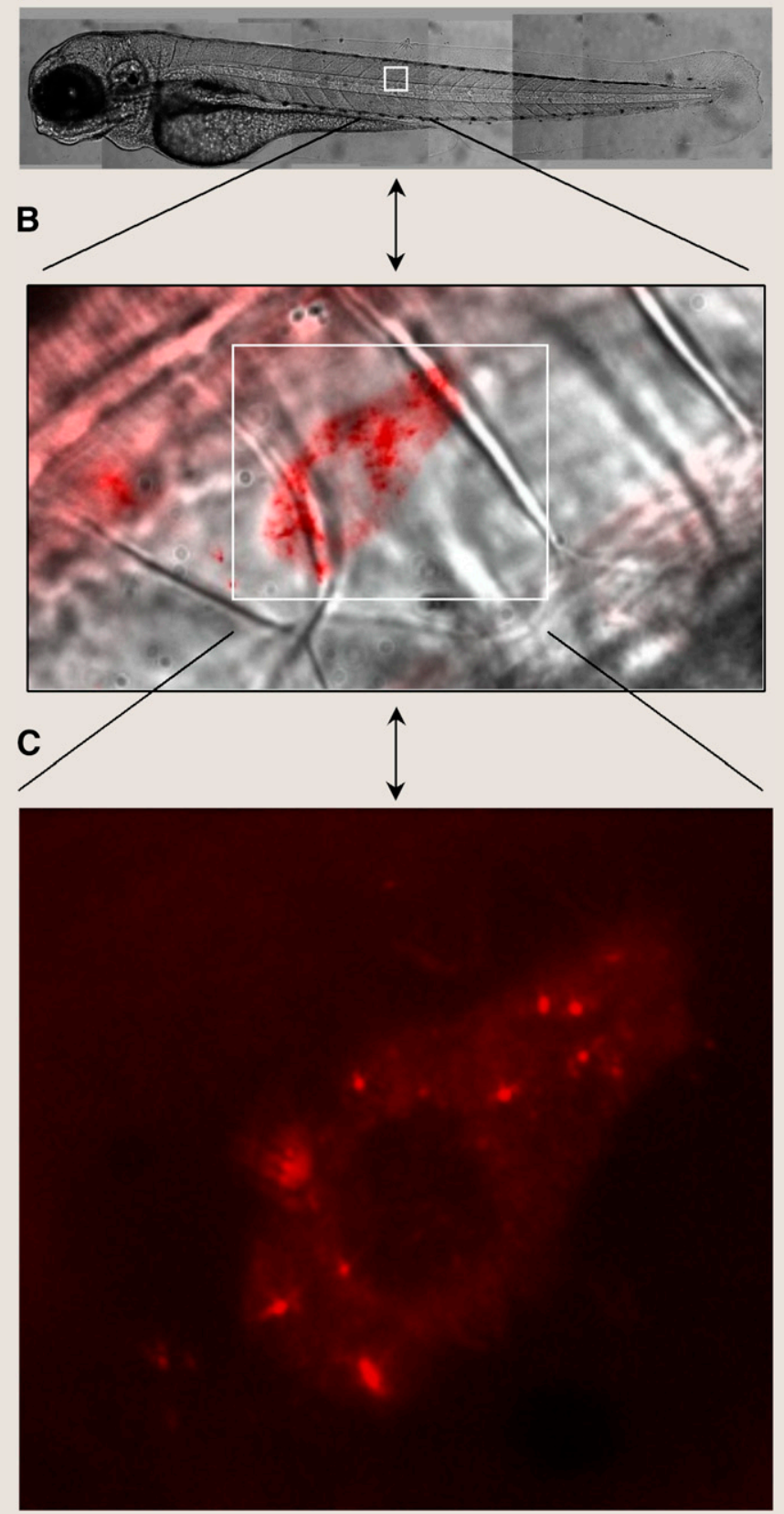

FIGURE 2. In vivo QD imaging in live zebrafish embryos. Two- to 4-cell-stage embryos were injected with red QDs and were allowed to develop to the larva stage. Live fish were observed in DIC (A and B) or epifluorescence (B and $C$ ) on an inverted microscope using a charge-coupled device camera. The brightness and photostability of QDs reveal the dynamic diffusion of single vesicles over a continuum of length scales from whole animals (millimeters) to subcellular levels (less than a micrometer). 
thetic efforts are under way to produce cadmium-free QD formulations while maintaining their unique photophysical properties. Examples of such cadmium-free QDs are InAs(x)P(1-x)/InP/ZnSe III-V-alloyed core or shell QDs (24). Another approach involves ternary structures such as CuInSe (25).

\section{CONCLUSION AND PERSPECTIVES}

Fluorescent QDs are now an integral part of our imaging toolbox. Although they will not likely replace MRI or PET, QDs can act in synergy in niche applications requiring high sensitivity, multiplexing, and better spatial and temporal resolution. Their potential use in the clinic for imaging of patients will be possible only when toxicity issues are fully understood and smaller sizes are achieved to enable clearance from the body. In the meantime, QDs are already opening exciting new avenues in small-animal imaging by bringing us closer to the ultimate goal of probing biologic systems at all length scales: from the level of the whole organism (macroscale) down to the cellular and molecular level (nanoscale) using a single probe (Fig. 2). Although QDs may never make it to the clinic, the development of QD imaging technologies in animals may shed new light on the basic molecular processes underlying disease in whole live organisms.

\section{ACKNOWLEDGMENTS}

We thank Professor Anna Wu for insightful discussions. Microscopy images in Figure 2 were obtained at the California NanoSystems Institute Advanced Light Microscopy/Spectroscopy Shared Facility at UCLA. This work was funded by the National Institutes of Health (EB000312-06A2) and by a fellowship from the Human Frontier Science Program.

\section{REFERENCES}

1. Debbage P, Jaschke W. Molecular imaging with nanoparticles: giant roles for dwarf actors. Histochem Cell Biol. 2008;130:845-875.

2. Bentolila LA, Doose S, Ebenstein Y, et al. Peptide-functionalized quantum dots for live diagnostic imaging and therapeutic applications. In: Mattoussi H, Cheon J, eds. Inorganic Nanoprobes for Biological Sensing and Imaging. Boston, MA: Artech House Publisher; 2009:45-69.

3. Liu W, Choi HS, Zimmer JP, Tanaka E, Frangioni JV, Bawendi M. Compact cysteine-coated $\mathrm{CdSe}(\mathrm{ZnCdS})$ quantum dots for in vivo applications. J Am Chem Soc. 2007;129:14530-14531.

4. Liu W, Howarth M, Greytak AB, et al. Compact biocompatible quantum dots functionalized for cellular imaging. J Am Chem Soc. 2008;130:1274-1284.
5. Gao X, Cui Y, Levenson RM, Chung LW, Nie S. In vivo cancer targeting and imaging with semiconductor quantum dots. Nat Biotechnol. 2004;22:969-976.

6. Cai W, Chen K, Li ZB, Gambhir SS, Chen X. Dual-function probe for PET and near-infrared fluorescence imaging of tumor vasculature. J Nucl Med. 2007;48:1862-1870.

7. Cai W, Shin DW, Chen K, et al. Peptide-labeled near-infrared quantum dots for imaging tumor vasculature in living subjects. Nano Lett. 2006;6:669-676.

8. Kim S, Lim YT, Soltesz EG, et al. Near-infrared fluorescent type II quantum dots for sentinel lymph node mapping. Nat Biotechnol. 2004;22:93-97.

9. Frangioni JV, Kim SW, Ohnishi S, Kim S, Bawendi MG. Sentinel lymph node mapping with type-II quantum dots. Methods Mol Biol. 2007;374:147-159.

10. So MK, Xu C, Loening AM, Gambhir SS, Rao J. Self-illuminating quantum dot conjugates for in vivo imaging. Nat Biotechnol. 2006;24:339-343.

11. Voura EB, Jaiswal JK, Mattoussi H, Simon SM. Tracking metastatic tumor cell extravasation with quantum dot nanocrystals and fluorescence emission-scanning microscopy. Nat Med. 2004;10:993-998.

12. Stroh M, Zimmer JP, Duda DG, et al. Quantum dots spectrally distinguish multiple species within the tumor milieu in vivo. Nat Med. 2005;11:678-682.

13. Michalet X, Pinaud FF, Bentolila LA, et al. Quantum dots for live cells, in vivo imaging, and diagnostics. Science. 2005;307:538-544.

14. Schipper ML, Cheng Z, Lee SW, et al. microPET-based biodistribution of quantum dots in living mice. J Nucl Med. 2007;48:1511-1518.

15. Schipper ML, Iyer G, Koh AL, et al. Particle size, surface coating, and PEGylation influence the biodistribution of quantum dots in living mice. Small. 2009;5:126-134.

16. Jin T, Yoshioka Y, Fujii F, Komai Y, Seki J, Seiyama A. Gd(3+)-functionalized near-infrared quantum dots for in vivo dual modal (fluorescence/magnetic resonance) imaging. Chem Commun (Camb). 2008;44:5764-5766.

17. Woodward JD, Kennel S, Mirzadeh S, et al. In vivo SPECT/CT imaging and biodistribution using radioactive $\mathrm{Cd}^{125 m} \mathrm{Te} / \mathrm{ZnS}$ nanoparticles. Nanotechnology. 2007; 18:175103.1-175103.5.

18. Giepmans BN, Deerinck TJ, Smarr BL, Jones YZ, Ellisman MH. Correlated light and electron microscopic imaging of multiple endogenous proteins using quantum dots. Nat Methods. 2005;2:743-749.

19. Choi HS, Liu W, Misra P, et al. Renal clearance of quantum dots. Nat Biotechnol. 2007;25:1165-1170.

20. Lovric J, Cho SJ, Winnik FM, Maysinger D. Unmodified cadmium telluride quantum dots induce reactive oxygen species formation leading to multiple organelle damage and cell death. Chem Biol. 2005;12:1227-1234.

21. Botsoa J, Lysenko V, Géloën A, Marty O, Bluet JM, Guillot G. Application of 3C-SiC quantum dots for living cell imaging. Appl Phys Lett. 2008;92:173902173903.

22. Warner JH, Hoshino A, Yamamoto K, Tilley RD. Water-soluble photoluminescent silicon quantum dots. Angew Chem Int Ed Engl. 2005;44:4550-4554.

23. Aharoni A, Mokari T, Popov I, Banin U. Synthesis of InAs/CdSe/ZnSe core/ shell1/shell2 structures with bright and stable near-infrared fluorescence. $J \mathrm{Am}$ Chem Soc. 2006;128:257-264.

24. Kim SW, Zimmer JP, Ohnishi S, Tracy JB, Frangioni JV, Bawendi MG. Engineering InAs(x)P(1-x)/InP/ZnSe III-V alloyed core/shell quantum dots for the near-infrared. J Am Chem Soc. 2005;127:10526-10532.

25. Allen PM, Bawendi MG. Ternary I-III-VI quantum dots luminescent in the red to near-infrared. J Am Chem Soc. 2008;130:9240-9241. 